\author{
${ }^{1}$ María Gloria Corbalán Carrillo \\ ${ }^{2}$ Óscar Hernández Vian \\ ${ }^{3}$ Montserrat Carré Catases \\ ${ }^{4}$ Georgina Paul Galcerán \\ ${ }^{5}$ Blanca Hernández Vian \\ ${ }^{3}$ Corpus Marzo Duque
}

${ }^{1}$ Institut Català de la Salut. Centro de Atención Primaria de Castellar del Vallès. ${ }^{2}$ Institut Català de la Salut. SAP Metropolitana Nord. ${ }^{3}$ Institut Català de la Salut. Centro de Atención Primaria Creu Alta. ${ }^{4}$ Institut Català de la Salut. Centro de Atención Primaria de Sant Quirze del Vallès. ${ }^{5}$ Institut Català de la Salut. Centro de Atención Primaria de la Concordia

\section{Sobrecarga, ansiedad y depresión en cuidadores de pacientes incluidos en el programa de atención domiciliaria}

\author{
Overload, anxiety and depression \\ in caregivers of patients included in \\ the bome care program
}

\author{
Correspondencia: \\ María Gloria Corbalán Carrillo \\ C/ Guerau de liost, 9 \\ Castellar del Valles 08211 \\ Teléf.: 630639491 \\ E-mail: gloria_coca@yahoo.es
}

\section{RESUMEN \\ El objetivo principal es determinar la sobrecarga, la prevalencia de ansiedad y depresión y su relación con la sobrecarga en cuidadores. Estudio descriptivo transversal. \\ Variables: edad, sexo, parentesco, patología del paciente, ansiedad y depresión del cuidador. Se utilizó la escala de Zarit para determinar el grado de sobrecarga. Se incluyeron 48 cuidadores y 48 pacientes. Se obtuvieron diferencias estadísticamente significativas entre sobrecarga y el sexo del cuidador $(\mathrm{p}=0,037)$. Un $87 \%$ de mujeres sufrían sobrecarga intensa. La prevalencia de ansiedad y depresión es menor que en otros estudios, aunque el grado de sobrecarga es mayor. La diferencia estadísticamente significativa puede deberse a que la mujer asume tradicionalmente el rol de cuidadora, mientras que los hombres buscan ayuda social antes. Este estudio ayudará a programar intervenciones para prevenir la sobrecarga, priorizando a las cuidadoras femeninas y de más edad.}

PALABRAS CLAVE

Sobrecarga, ansiedad, depresión, cuidadores.

\begin{abstract}
SUMMARY
The main objective is to determine the degree of burden of caregivers, describe the prevalence of anxiety and depression and the relationship among these pathologies and burden. Descriptive cross-sectional study. Variables: age, sex, relationship, patient's diseases, caregiver's anxiety and depression. We use the Zarit scale to determine the degree of burden. 48 caregivers and 48 patients were included. Statistical significant differences were obtained between burden and caregiver's sex $(p=0.037)$. Women have greater burden (87\%). Anxiety and depression's prevalence is smaller than other studies. The significant difference between burden and sex can be due to the traditional caregiver's rol among women. (Men search social help before.) The burden degree is bigger than other studies. This study can help to develop intervention programs to prevent burden and to give priority to female and age caregivers.
\end{abstract}

\section{KEY WORDS}

Burden, anxiety, depression, caregivers.

\section{INTRODUCCIÓN}

El gradual envejecimiento de la población y la mayor supervivencia de las personas con enfermedades crónicas y discapacitantes ha propiciado el aumento de pacientes que precisan de cuidados (1).

Las personas que prestan estos cuidados pueden sufrir una sobrecarga que puede incapacitarlos para cuidar y afectar su salud, tanto física como mental. El concepto de sobrecarga se refiere a cómo los cuidadores, por el hecho de cuidar, perciben cómo su salud, vida social y personal, y situación económica cambian (1). Cuidar puede generar problemas emocionales o trastornos psiquiátricos, como la ansiedad y la depresión, en el cuidador.

Una de las patologías que más precisan de cuidados son las demencias, con una prevalencia de 1,5\% en las personas entre 60-69 años y hasta un $40 \%$ en nonagenarios (2). El deterioro cognitivo y funcional de las demencias produce un gran impacto en la familia y, sobre todo, en el cuidador principal, que es la persona que asume la responsabilidad total de la atención y el apoyo diario del paciente dependiente (3).
El cuidado de un paciente con demencia genera mayor estrés que el de un anciano con patología física, por lo que son un grupo de riesgo para el desarrollo de sobrecarga (4).

La finalidad de este estudio es conocer el perfil del cuidador y del paciente incluido en el programa de atención domiciliaria (ATDOM) de un centro de salud del Vallés Occidental (Barcelona), analizar la sobrecarga y sus posibles diferencias entre cuidadores de demencias y de otras patologías, y conocer la prevalencia de trastornos psicoafectivos y su probable relación con la sobrecarga.

\section{MÉTODOLOGÍA}

Se presenta un estudio descriptivo transversal:

Población: cuidadores y pacientes incluidos en el programa ATDOM. Los datos se recogieron a través de la historia clínica informatizada. 
Criterios de exclusión: cuidadores asalariados, sin la escala de Zarit (Anexo 1) realizada y cuidadores que pertenecieran a otro centro, por la imposibilidad de obtener sus datos clínicos.

Variables sociodemográficas: edad, sexo (paciente y cuidador) y parentesco. Otras variables: tipo de patología del paciente (demencia o no demencia), patología (ansiedad o depresión ) del cuidador.

Para medir la sobrecarga del cuidador se utilizó el cuestionario de Zarit, que presenta una sensibilidad del $84,6 \%$ y una especificidad del $85,3 \%$ (en los puntos de corte de 46-47) y una sensibilidad de $87,7 \%$ y especificidad de $94,2 \%$ para discriminar entre sobrecarga leve e intensa (5). El test de Zarit es una herramienta validada, frable, fácil de utilizar y de amplio uso internacional (6-7). La escala de Zarit mide la sobrecarga subjetiva, definida como las actitudes y reacciones emocionales ante la experiencia de cuidar. Consta de 22 ítems que se responden en escala de Likert de cinco puntos: nunca $=0$, rara vez $=1$, algunas veces $=2$, muchas veces $=3$, casi siempre $=4$ (8). La puntuación $<46$ es No sobrecarga. 46-47 a 55-56 Sobrecarga leve y >56 Sobrecarga intensa.

Las puntuaciones de sobrecarga se correlacionan significativamente con los síntomas psicopatológicos en el cuidador y con su salud física, así como con la relación entre cuidador y paciente (8).
El análisis de los datos se llevó a cabo con el programa SPSS V17.

Para establecer la relación de las variables cuantitativas se ha utilizado la prueba de $t$ Student o $U$-Mannwitney. Para las variables cualitativas la prueba de chi-cuadrado o test exacto de Fisher. Para la correlación entre variables se utilizó el test de Spearman.

Dentro de las consideraciones éticas se ha procedido a ocultar cualquier dato que pudiera identificar a los participantes en el estudio.

No es necesario recoger el consentimento informado ya que se han recogido los datos directamente de la historia clínica informatizada (ECAP).

\section{RESULTADOS}

Pacientes: $\mathrm{N}=48$

Sexo: mujeres, 72,9\%; hombres, $27,1 \%$

Edad: 86,02 (DE: 8,72)

Patología del paciente: No demencias, $72,9 \%$.

Demencias, $27,1 \%$

Cuidadores: $\mathrm{N}=48$

Sexo: mujeres, 70,8\%. Hombres, 29,2\%

Edad: 64,75 (DE: 12,20)

Parentesco: pareja, 31,3\%. Hijo-a, 56,3\%; otros familiares, $12,5 \%$

\begin{tabular}{|c|c|c|}
\hline \multicolumn{3}{|c|}{ Anexo 1. Escala de sobrecarga del cuidador de Zarit (Caregiver Burden Interview) } \\
\hline Ítem & Pregunta a realizar & Puntuación \\
\hline 1 & ¿Siente que su familiar solicita más ayuda de la que realmente necesita? & \\
\hline 2 & ¿Siente que debido al tiempo que dedica a su familiar ya no dispone de tiempo suficiente para usted? & \\
\hline 3 & ¿Se siente tenso cuando tiene que cuidar a su familiar y atender además otras responsabilidades? & \\
\hline 4 & ¿Se siente avergonzado por la conducta de su familiar? & \\
\hline 5 & ¿Se siente enfadado cuando está cerca de su familiar? & \\
\hline 6 & ¿Cree que la situación actual afecta de manera negativa a su relación con amigos y otros miembros de su familia? & \\
\hline 7 & ¿Siente temor por el futuro que le espera a su familiar? & \\
\hline 8 & ¿Siente que su familiar depende de usted? & \\
\hline 9 & ¿Se siente agobiado cuando tiene que estar junto a su familiar? & \\
\hline 10 & ¿Siente que su salud se ha resentido por cuidar a su familiar? & \\
\hline 11 & ¿Siente que no tiene la vida privada que desearía debido a su familiar? & \\
\hline 12 & ¿Cree que su vida social se ha visto afectada por tener que cuidar de su familiar? & \\
\hline 13 & ¿Se siente incómodo para invitar amigos a casa, a causa de su familiar? & \\
\hline 14 & ¿Cree que su familiar espera que usted le cuide, como si fuera la única persona con la que puede contar? & \\
\hline 15 & ¿Cree que no dispone de dinero suficiente para cuidar a su familiar además de sus otros gastos? & \\
\hline 16 & ¿Siente que será incapaz de cuidar a su familiar por mucho más tiempo? & \\
\hline
\end{tabular}


Corbalán Carrillo, M. ${ }^{a}$ G.; Hernández Vian, O.; Carré Catases, M.; Paul Galceran, G.; Hernández Vian, B.; Marzo Duque, C.: SOBRECARGA, ANSIEDAD Y DEPRESIÓN EN CUIDADORES DE PACIENTES INCLUIDOS EN EL PROGRAMA DE ATENCIÓN DOMICILIARIA

\begin{tabular}{|c|c|c|}
\hline \multicolumn{3}{|c|}{ Anexo 1. (Continuación) Escala de sobrecarga del cuidador de Zarit (Caregiver Burden Interview) } \\
\hline Ítem & Pregunta a realizar & Puntuación \\
\hline 17 & ¿Siente que ha perdido el control sobre su vida desde que la enfermedad de su familiar se manifestó? & \\
\hline 18 & ¿Desearía poder encargar el cuidado de su familiar a otras personas? & \\
\hline 19 & ¿Se siente inseguro acerca de lo que debe hacer con su familiar? & \\
\hline 20 & ¿Siente que debería hacer más de lo que hace por su familiar? & \\
\hline 21 & ¿Cree que podría cuidar de su familiar mejor de lo que lo hace? & \\
\hline 22 & En general: ¿Se siente muy sobrecargado por tener que cuidar de su familiar? & \\
\hline
\end{tabular}

Cada ítem se valora así:

Puntuación de cada ítem (sumar todos para el resultado):

\begin{tabular}{|lc|}
\hline Frecuencia & Puntuación \\
Nunca & 0 \\
Casi nunca & 1 \\
A veces & 2 \\
Bastantes veces & 3 \\
Casi siempre & 4 \\
\hline
\end{tabular}

Puntuación máxima de 88 puntos. No existen normas ni puntos de corte establecidos. Sin embargo, suele considerarse indicativa de "no sobrecarga" una puntuación inferior a 46, y de "sobrecarga intensa" una puntuación superior a 56.

Patología del cuidador: no patología, 62,5\%. Ansiedad, 10,4\%. Trastornos mixtos de ansiedad y depresión, 18,8\%. Depresión: 8,3\%.

Puntuación media del test de Zarit: 57 (DE: 18,36). Puntuación media Zarit en mujeres: 59,76 (DE: 19,15); en hombres: 50,29 (DE: 14,79).

Test de Zarit: No sobrecarga, 29,2\%. Sobrecarga leve, 22,9\%. Sobrecarga intensa, 47,9\%.

Se ha encontrado asociación estadísticamente significativa entre las variables sobrecarga en el test de Zarit y sexo del cuidador $(\mathrm{P}=0,037)$. De los cuidadores que presentaban sobrecarga intensa, un $87 \%$ eran mujeres, mientras que un $13 \%$ eran hombres.

Para el análisis de los datos se ha realizado una descriptiva de las variables principales (Tabla 1 ).

Las asociaciones: edad paciente-sobrecarga y edad cuidador sobrecarga se han analizado con el test Kruskal-Wallis.

Tampoco se encontró relación estadísticamente significativa entre patología paciente y patología cuidador $(\mathrm{p}=0,463)$.

\section{DISCUSIÓN}

La edad de los pacientes es más elevada que en otros estudios (3, 9, 10), predominando el sexo femenino $(3,9,10)$. Este dato puede deberse a la mayor esperanza de vida de la mujer en nuestro país.

La edad de los cuidadores también es más elevada que en otros estudios y la gran mayoría son mujeres $(1,3,4,8,10,11,12)$. Estos datos concuerdan con la imagen tradicional de la mujer como cuidadora. Diversos estudios sitúan esta preponderancia de la mujer como cuidadora entre un $60 \%$ y un $87,4 \%$ (1).

Hay un predominio de los cuidadores que son hijos/as del paciente, seguidos de la pareja, como ya se ha constatado en otros estudios $(1,3,10,12)$.

La prevalencia de la ansiedad y depresión es menor que en otros estudios $(1,3,8)$. Creemos que este dato se debe a los diferentes instrumentos de medida utilizados en los diferentes estudios. En nuestro caso, se utilizó el diagnóstico sin realizar escalas para la valoración de la depresión o la ansiedad.

El grado de sobrecarga es algo más elevado que en otros estudios (1, 10,11).

La relación significativa entre sobrecarga y sexo ( las mujeres presentan una mayor sobrecarga) puede derivar del hecho de que la mujer asume tradicionalmente el rol de cuidar, mientras que el hombre, que no ha desarrollado habitualmente este papel, busca más mecanismos de ayuda social y más tempranamente $(13,14)$. 
Corbalán Carrillo, M. ${ }^{a}$ G.; Hernández Vian, O.; Carré Catases, M.; Paul Galceran, G.; Hernández Vian, B.; Marzo Duque, C.: SOBRECARGA, ANSIEDAD Y DEPRESIÓN EN CUIDADORES DE PACIENTES INCLUIDOS EN EL PROGRAMA DE ATENCIÓN DOMICILIARIA

\begin{tabular}{|c|c|c|c|c|c|}
\hline & & \multicolumn{4}{|c|}{ Medianas } \\
\hline & & No sobrecarga & Leve & Intensa & p-valor \\
\hline Edad paciente & & 85,71 (DE:7,07) & $84,91(\mathrm{DE}: 16,08)$ & 86,74(DE:9,4) & 0,594 \\
\hline \multirow[t]{3}{*}{ Edad cuidador } & & 66,14(DE:9,7) & 63,91(DE:11,80) & 64,4,3(DE:11,8) & 0,693 \\
\hline & & \multicolumn{4}{|c|}{ Porcentajes } \\
\hline & & No sobrecarga & Leve & Intenso & p-valor \\
\hline \multirow[t]{2}{*}{ Sexo paciente } & Mujer & $71,4 \%$ & $63,6 \%$ & $78,3 \%$ & \multirow{2}{*}{0,463} \\
\hline & Hombre & $28,6 \%$ & $36,4 \%$ & $21,7 \%$ & \\
\hline \multirow[t]{2}{*}{ Sexo cuidador } & Mujer & $64,3 \%$ & $45,5 \%$ & $87 \%$ & \multirow{2}{*}{0,037} \\
\hline & Hombre & $35,7 \%$ & $54,5 \%$ & $13 \%$ & \\
\hline \multirow[t]{2}{*}{ Patología paciente } & No demencias & $78,6 \%$ & $63,6 \%$ & $73,9 \%$ & \multirow{2}{*}{0,698} \\
\hline & Demencias & $21,4 \%$ & $36,4 \%$ & $26,1 \%$ & \\
\hline \multirow[t]{4}{*}{ Salud mental cuidador } & No patología & $85,7 \%$ & $63,6 \%$ & $47,8 \%$ & \multirow{4}{*}{0,449} \\
\hline & Ansiedad & $7,1 \%$ & $9,1 \%$ & $13 \%$ & \\
\hline & Ansiedad y depresión & $7,1 \%$ & $18,2 \%$ & $26,1 \%$ & \\
\hline & Depresión & $0 \%$ & $9,1 \%$ & $13,0 \%$ & \\
\hline \multirow[t]{3}{*}{ Parentesco } & Esposa & $35,7 \%$ & $35,7 \%$ & $21,7 \%$ & \multirow{3}{*}{0,389} \\
\hline & Hija & $42,9 \%$ & $42,9 \%$ & $69,6 \%$ & \\
\hline & Otros & $21,4 \%$ & $21,4 \%$ & $8,7 \%$ & \\
\hline
\end{tabular}

\section{BIBLIOGRAFÍA}

1. Lara PG, González PAA, Blanco LLA. Perfil del cuidador: sobrecarga y apoyo familiar e institucional del cuidador primario en el primer nivel de atención. Rev Esp Med Quir 2008; 13: 159-66.

2. Qiu Chengxuan, De Ronchi Diana, Fratiglioni Laura. The epidemiology of the dementias:an Update. Curr Opin Psychiatry 2007; 20; 380-5.

3. Domínguez JA, Ruiz M, Gómez J, Gallego E, Valero J, Izquierdo M.T. Ansiedad y depresión en cuidadores de pacientes dependientes. Semergen 2012; 38: $16-23$.

4. Méndez L, Giraldo O, Aguirre- Acevedo D, Lopera F. Relación entre ansiedad, depresión, estrés y sobrecarga en cuidadores familiares con demència tipo Alzheimer por mutación e280a en presenilina 1. Rev Chil Neuropsicol 2010; 5 : $137-45$.

5. Molina JM, Velasco MA. El apoyo social como modulador de la carga del cuidador de enfermos de Alzheimer. Psicología y Salud. 2005; 15 (1): 33-43.

6. Van Durme T, Macq J, Jeanmart C, Gobert M.Tools for measuring the impact of informal caregiving of the elderly: A literatura review. Int J Nurs 2012; 49 (4): 490-504.

7. Seng BK, Luo N, Ng WY, Lim J, Chionh HL, Goh J, Yap P.Validity and reliability of the Zarit Burden Interview in assessing caregiving burden. Ann Acad Med Singapore 2010; 39: 758-63.

8. Alfaro-Ramírez del Castillo OI, Morales- Vigil T, Vázquez - Pineda F, SánchezRomán S, Ramos del Río B, Guevara López U. Sobrecarga, ansiedad y depre- sión en cuidadores primarios de pacientes con dolor crónico y terminales. Rev Med Inst Mex Seguro Soc 2008; 46: 485-94.

9. Instituto de Salud Carlos III. Visita domiciliaria programada de enfermería a persones mayores de 65 años. Madrid: Instituto de salud Carlos III, 2002.

10. Yonte Huete F, Urién Pérez Z, Martín Gutiérrez M, Montero Herrero MR. Sobrecarga del cuidador principal. Nure Investigación, no 48 , SeptiembreOctubre 10 .

11. Leggett A, Zarit $s$, Taylor A, Galvin JE. Stress and Burden among caregivers of patients with Lewy body dementia. The Gerontologist 2010; 51: 76-85.

12. Garrido R, Menezes PR. Impact on caregivers of elderly patients with dementia treated at a psychogeriatric service. Rev Saude Publica 2004; 38: 835-41. Epub 2004 Dec 10.

13. Zabalegui Yanoz A, Juandó Prats C, Saénz de Ormijana Hernández A y cols. Los cuidadores informales en España: perfil y cuidados prestados. Revista Rol de enfermería 2007; 30: 513-8.

14. Paleo Díaz N, Falcón Rodríguez N, Rodríguez Paleo L. Sobrecarga de los cuidadores de pacientes con demencia de Alzheimer. [Disponible en internet]. Disponible en: http://weblog.maimonides.edu/gerontologia2004/ archives/000919.html Consulta: mayo 27 de 2010.

15. Ferré Grau C, Rodero Sánchez V, Vives Relats C, Cid Buera D. El Mundo del cuidador familiar. Tarragona: Silva Editorial, 2008. 